\title{
Bio-Functionalized Magnetic Nanoparticles for the Immunoassay of C-Reactive Protein and Procalcitonin in Cervicovaginal Secretions of Pregnant Women with Preterm Prelabor Rupture of Membranes to Predict Early-Onset Neonatal Sepsis
}

\author{
Sau Xiong Ang $\mathbb{D}^{\prime}$ \\ Chie-Pein Chen' \\ Fang-Ju Sun ${ }^{2}$ \\ Chen-Yu Chen $\mathbb{D}^{1,3}$ \\ 'Department of Obstetrics and \\ Gynecology, Mackay Memorial Hospital, \\ Taipei, Taiwan; ${ }^{2}$ Department of Medical \\ Research, Mackay Memorial Hospital, \\ Taipei, Taiwan; ${ }^{3}$ Department of Medicine, \\ Mackay Medical College, New Taipei City, \\ Taiwan
}

Correspondence: Chen-Yu Chen Department of Obstetrics and Gynecology, Mackay Memorial Hospital, No. 92, Section 2, Zhong-Shan North Road, Taipei, 10449, Taiwan

Tel +886-2-2543-3535

Fax +886-2-2543-3642

Email fl2248I@mmh.org.tw
Purpose: Early-onset sepsis is a major cause of neonatal morbidity and mortality. C-reactive protein (CRP) and procalcitonin (PCT) are acute phase reactants related to infection. The aim of this study was to explore the feasibility of measuring CRP and PCT concentrations in cervicovaginal secretions of pregnant women with preterm prelabor rupture of membranes (PPROM) using an immunomagnetic reduction (IMR) assay to predict early-onset neonatal sepsis.

Patients and Methods: This prospective study was performed at Mackay Memorial Hospital, Taipei, Taiwan from February 2015 to January 2018. Pregnant women with PPROM between 22 and 34 weeks of gestation were recruited. CRP and PCT concentrations in cervicovaginal secretions were measured using an IMR assay.

Results: Thirty-five cervicovaginal secretion samples were obtained. After excluding two neonatal deaths, early-onset neonatal sepsis was diagnosed in 15 of the 33 surviving neonates. There was no significant relationship between cervicovaginal secretion CRP level and neonatal sepsis; however, cervicovaginal secretion PCT levels were significantly higher in the neonatal sepsis group than in the non-sepsis group ( $45.99 \mathrm{vs} 9.54 \mathrm{ng} / \mathrm{mL}, P=0.039$ ). Receiver operating characteristic (ROC) curve analysis revealed a PCT cut-off level of $20.60 \mathrm{ng} / \mathrm{mL}$ to predict earlyonset sepsis, and the area under the ROC curve was 0.71 ( $95 \%$ confidence interval 0.52 to 0.90 , $P=0.039$ ), with sensitivity and specificity of $73.3 \%$ and $77.8 \%$, respectively.

Conclusion: Measuring the concentration of PCT in cervicovaginal secretions with an IMR assay can predict early-onset sepsis in neonates born to mothers with PPROM.

Keywords: C-reactive protein, procalcitonin, immunomagnetic reduction, magnetic nanoparticle, preterm prelabor rupture of membranes, early-onset neonatal sepsis

\section{Introduction}

Preterm prelabor rupture of membranes (PPROM) is defined as the spontaneous rupture of fetal membranes before the onset of labor by 37 weeks of gestation. PPROM has been reported to occur in approximately $3 \%$ of pregnancies and to account for $30 \%$ of preterm births. ${ }^{1}$ Approximately $30 \%$ of mothers with PPROM develop chorioamnionitis, which can subsequently lead to a significantly higher rate of neonatal morbidity. ${ }^{2}$ Previous studies have demonstrated associations between both histologic and clinical chorioamnionitis with neonatal sepsis. ${ }^{3}$ However, 
chorioamnionitis is practically sensitive but lacks specificity for the diagnosis of neonatal sepsis, particularly among preterm neonates. ${ }^{4}$ Early-onset sepsis remains a major cause of morbidity and mortality in preterm neonates, ${ }^{5}$ and thus identifying the neonates who will develop early-onset sepsis is crucial.

Previous studies have demonstrated that various potential biomarkers can be used to effectively screen for neonatal sepsis, including $\mathrm{C}$-reactive protein (CRP) and procalcitonin (PCT). ${ }^{6,7} \mathrm{CRP}$ and PCT are acute phase reactants which are widely used in current clinical practice to detect systemic inflammation and infection. CRP is synthesized in the liver in response to proinflammatory cytokines (interleukin- $1 \beta[\mathrm{IL}-$ $1 \beta]$, IL-6, and tumor necrosis factor [TNF]), and it is released into the systemic circulation after inflammation or infection. ${ }^{8}$ PCT, the prohormone of calcitonin, is limited to thyroid neuroendocrine cells, and it is not released into the systemic circulation before cleavage into calcitonin. ${ }^{9}$ When systemic inflammation is caused by bacterial infection, PCT synthesis is triggered by endotoxins and pro-inflammatory cytokines (such as IL-1 $1 \beta$, IL-6 and TNF) in nearly all peripheral tissues, and is then released into the systemic circulation. ${ }^{10}$

CRP and PCT are commonly measured by enzyme-linked immunosorbent assay (ELISA) or immunoturbidimetry. ${ }^{11,12}$ However, ELISA is time-consuming and suboptimal color signal intensity measurements are possible due to crossreaction with bound antibodies, while bilirubin, hemoglobin and intrafat can cause interference in immunoturbidimetry measurements. The immunomagnetic reduction (IMR) assay was developed to overcome these limitations, and it can be used to identify biomolecules using bio-functionalized magnetic nanoparticles. IMR assays have been shown to be able to detect biomarkers in body fluids with ideal specificity and sensitivity. ${ }^{13-16}$

Few previous studies have evaluated the relationships between CRP or PCT in cervicovaginal secretions of pregnant women with PPROM and infection, and the results have been inconsistent. Furthermore, few studies have focused on early-onset neonatal sepsis. ${ }^{17-19}$ However, accurate and timely antepartum identification of earlyonset neonatal sepsis can allow for early interventions after childbirth and also avoid unnecessary neonatal exposure to antibiotics, which can improve neonatal prognosis and outcomes. ${ }^{20}$ Therefore, we conducted this pilot study to measure CRP and PCT concentrations in cervicovaginal secretions of pregnant women with PPROM using an IMR assay, and further investigate the relationships between these two biomarkers and early-onset neonatal sepsis.

\section{Materials and Methods}

\section{Sample Collection}

We performed this prospective study at the delivery room of Mackay Memorial Hospital, Taipei, Taiwan from February 2015 to January 2018, and enrolled pregnant women with PPROM between 22 and 34 weeks of gestation. PPROM was diagnosed according to clinical assessment and laboratory findings, such as amniotic fluid pooling in the vagina, a positive nitrazine test of vaginal fluid, decreased amniotic fluid volume in an ultrasonographic examination, and commercial tests for amniotic proteins. ${ }^{1}$ All of the patients received prophylactic antibiotics with a combination of intravenous ampicillin and erythromycin followed by oral amoxicillin and erythromycin. ${ }^{1}$ Maternal serum white blood cell (WBC) count and CRP level were routinely measured before administering the antibiotics. Cervicovaginal secretions were collected using a sterile cotton swab inserted into the vaginal posterior fornix for 10 seconds during a speculum examination. We then inserted the swab tip into a plastic tube containing $3 \mathrm{~mL}$ of phosphate buffered saline (PBS) and left it in place for 10 seconds to allow the swab to become saturated. The samples were then sent to our laboratory for CRP and PCT measurements using an IMR analyzer (XacPro-E, MagQu, New Taipei City, Taiwan). Chorioamnionitis was diagnosed according to either clinical or histological criteria. ${ }^{21,22}$ The clinical criteria were a maternal temperature $>38.0^{\circ} \mathrm{C}$ and fundal tenderness on physical examination; and the histological criteria were acute chorioamnionitis or funisitis verified in placental pathology. Early-onset neonatal sepsis was defined based on a positive blood culture or the presence of clinical signs or symptoms (such as fever, shortness of breath, neurologic signs not explained by other conditions, pneumonia patch on a chest $\mathrm{X}$-ray, pleocytosis in cerebrospinal fluid or laboratory findings of bandemia, and elevated CRP levels) within 72 hours of delivery. ${ }^{3,5}$ This study was reviewed and approved by the Institutional Review Board of Mackay Memorial Hospital (approval no. 09MMHIS056). All participants were informed about the purpose of the study, and they all signed informed consent forms. This study was conducted in accordance with the Declaration of Helsinki, and all personal identifiers were anonymized prior to analysis.

\section{Preparation of Magnetic Reagents}

Figure 1A shows a photograph of the magnetic nanoparticles (MagQu, Ltd, New Taipei City, Taiwan) taken using a scanning electron microscope (JSM-6700F, JEOL, Tokyo, Japan), which was acquired under dehydrated 
A

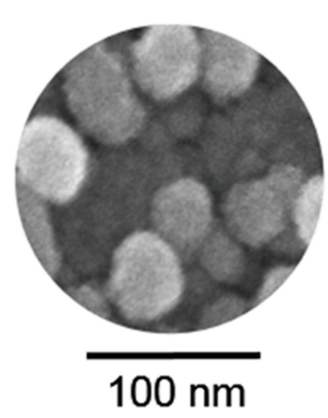

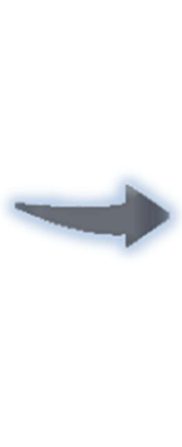

B

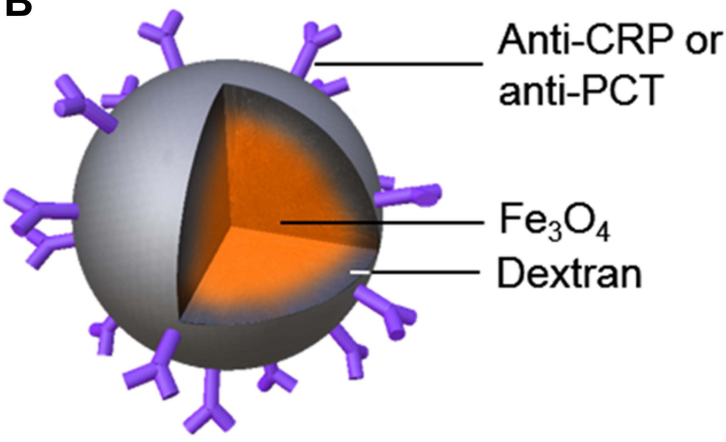

Figure I Depiction of magnetic nanoparticles. (A) A photograph of nanoparticles under dehydrated condition by scanning electron microscope (I00,000X). (B) Illustration of a magnetic $\mathrm{Fe}_{3} \mathrm{O}_{4}$ nanoparticle coated with dextran and anti-CRP or ant-PCT antibodies.

Abbreviations: CRP, C-reactive protein; PCT, procalcitonin.

conditions. Figure 1B shows an illustration of a magnetic $\mathrm{Fe}_{3} \mathrm{O}_{4}$ nanoparticle covered with dextran as a hydrophilic surfactant and anti-CRP (MAB17071-500, R\&D Systems, Minneapolis, USA) or anti-PCT (NB120-14817, Novus Biologicals, Colorado, USA) antibodies.

\section{Mechanism of the IMR Assay}

Figure 2 shows illustrations of interactions between antigens (CRP or PCT) and antibodies (anti-CRP or anti-PCT) in an IMR assay. Before antigen-antibody reactions, individual homogeneous magnetic nanoparticles coated with antibodies were thoroughly dispersed in PBS solution $(\mathrm{pH}=7.2)$. These magnetic nanoparticles were induced to oscillate and rotate by external multiple alternating current (AC) magnetic fields (Figure 2A). After antigen-antibody reactions, the magnetic nanoparticles connected with the antigens via the antibodies coated on the shell surface and became larger and clustered, subsequently causing them to spin much slower than the original individual magnetic nanoparticles (Figure 2B). The reaction time from the beginning of antibody-antigen association to the end of antibody-antigen dissociation took about 3 to 4 hours. The final IMR signal was obtained by measuring the attenuation in the percentage of $\mathrm{AC}$ magnetic susceptibility of the magnetic reagent, which was calculated using the equation: IMR $(\%)=\left(\chi_{\mathrm{ac}, \mathrm{o}}-\chi_{\mathrm{ac}, \phi}\right) / \chi_{\mathrm{ac}, \mathrm{o}} \times 100 \%$, where $\chi_{\mathrm{ac}, \mathrm{o}}$ is the mixed-frequency $\mathrm{AC}$ magnetic susceptibility before antigen-antibody reactions, $\chi_{\mathrm{ac}, \phi}$ is the mixed-frequency AC magnetic susceptibility after antigen-antibody reactions, and $\phi$ is the concentration of CRP or PCT. ${ }^{13}$

\section{Establishment of Calibration Curves}

CRP (C4063, Sigma-Aldrich, Saint Louis, USA) and PCT (SRP6003, Sigma-Aldrich, Saint Louis, USA) antigens were used to establish calibration curves of the IMR signals. The mean value and standard deviation of each concentration were obtained through duplicate measurements of IMR signals. Calibration curves were calculated using the four-parameter logistic equation: $\operatorname{IMR}(\%)=(\mathrm{A}-\mathrm{B}) /\left[1+\left(\phi_{\mathrm{CRP} \text { or PCT }} / \phi_{\mathrm{o}}\right)^{\mathrm{r}}\right]+\mathrm{B}$, where $\mathrm{A}$ is the minimal IMR signal, $\mathrm{B}$ is the maximal IMR signal, $\phi_{\mathrm{CRP}}$ or PCT is the CRP or PCT concentration, $\phi_{\mathrm{o}}$ is the
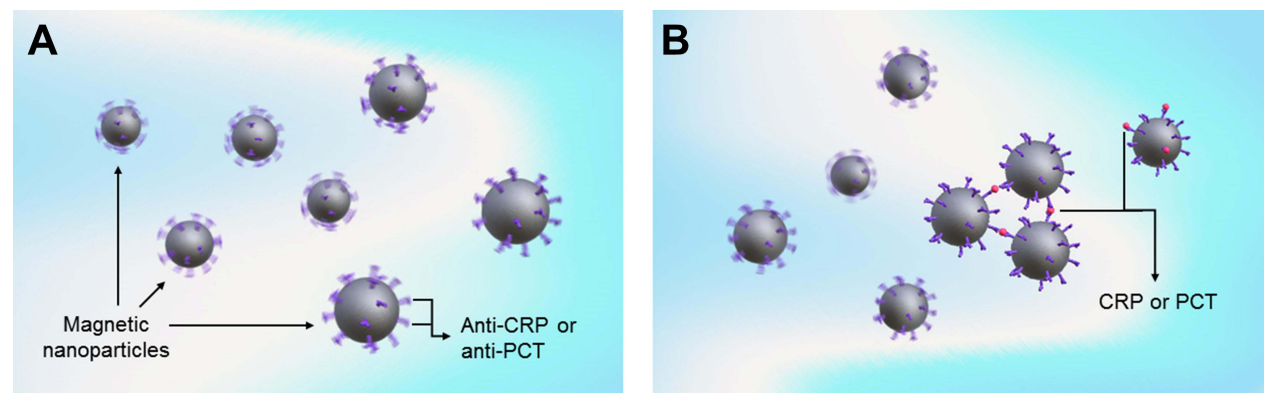

Figure 2 Illustration of the association between CRP and PCT and magnetic nanoparticles coated with anti-CRP or anti-PCT antibodies in the IMR assay. (A) Under the applied external alternating current magnetic fields, the magnetic nanoparticles oscillated and spun individually. (B) The magnetic nanoparticles became larger and clustered after binding with CRP or PCT, and thus oscillated and spun much slower than the original individual magnetic nanoparticles.

Abbreviations: CRP, C-reactive protein; PCT, procalcitonin; IMR, immunomagnetic reduction. 
A

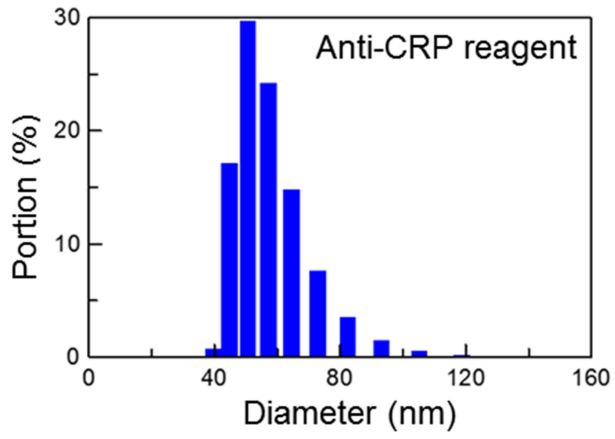

B

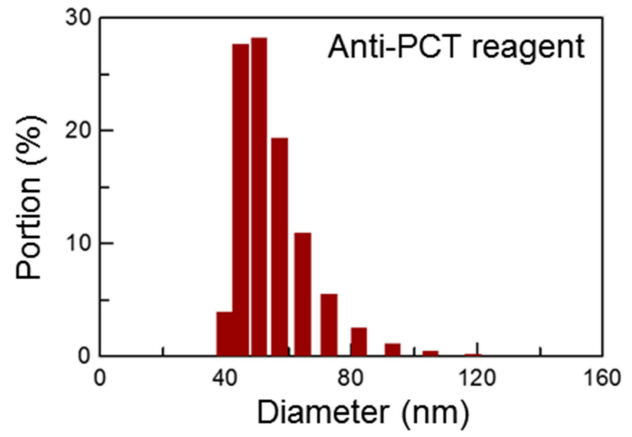

Figure 3 Magnitude and distribution of the anti-CRP and anti-PCT magnetic reagents determined by dynamic laser scattering. (A) The reagent with anti-CRP antibodies. (B) The reagent with anti-PCT antibodies.

Abbreviations: CRP, C-reactive protein; PCT, procalcitonin.

concentration of the inflection point corresponding to the half maximal IMR signal, and $r$ is the slope at the inflection point of the calibration curve. ${ }^{13}$

\section{Statistical Analysis}

SPSS version 26.0 (IBM Corporation, Armonk, NY, USA) was used for the statistical analyses. Spearman correlation analysis was used to evaluate correlations between detected biomarkers. The Kolmogorov-Smirnov test was used to determine whether continuous variables were normally distributed. The Student's $t$-test was used for normally distributed data; otherwise, the Mann-Whitney $U$-test was used. Receiver operating characteristic (ROC) curve analysis was used to identify the optimum cut-off value to predict early-onset neonatal sepsis. A $P$ value $<$ 0.05 was considered to be statistically significant.

\section{Results}

\section{Magnetic Reagents}

Figure 3 shows the magnitude and distribution of the magnetic nanoparticles analyzed using dynamic laser scattering (Nanotrac 150, Microtrac, USA). The reagent with anti-CRP antibodies was referred to as the CRP reagent, and the reagent with anti-PCT antibodies was referred to as the PCT reagent. The mean diameters of the magnetic nanoparticles in the CRP and PCT reagents were 53.6 $\pm 10.6 \mathrm{~nm}$ (Figure 3A) and 51.0 \pm $10.5 \mathrm{~nm}$ (Figure 3B), respectively. The magnetic concentration of the reagent was $0.1 \mathrm{emu} / \mathrm{g}$, which was stored at $4^{\circ} \mathrm{C}$.

\section{Calibration Curves of the IMR Signals}

Table 1 and Figure 4 show the data and calibration curves of CRP and PCT concentration-dependent IMR signals. The IMR signal for zero concentration of a biomarker denotes the background level of the IMR signal. Of note, the IMR signal for a CRP or PCT concentration of $0.01 \mathrm{ng} / \mathrm{mL}$ was significantly higher than the background level. The IMR signals increased as the concentrations of both biomarkers increased, except for a CRP concentration of $1000 \mathrm{ng} / \mathrm{mL}$ (Figure 4A). The decrease in IMR signal for a CRP concentration of 1000 $\mathrm{ng} / \mathrm{mL}$ revealed that the Hook effect occurred for a CRP concentration higher than $300 \mathrm{ng} / \mathrm{mL}$, and hence data for a CRP concentration higher than $300 \mathrm{ng} / \mathrm{mL}$ were not included in the analysis. For the calibration curves of PCT measurements, the Hook effect was not observed for a concentration from 0.01 to $500 \mathrm{ng} / \mathrm{mL}$ (Figure 4B).

In the logistic equation for the calibration curve, parameter A denotes the noise level for the IMR signal, which was primarily due to electronic interactions of the AC magnetosusceptometer. The limit of detection (LOD) was defined as a concentration higher than the noise level by three standard deviations for IMR signals at the lowest detectable concentration. In this experiment, the standard deviations of IMR signals at a low concentration $(0.01 \mathrm{ng} / \mathrm{mL})$ were $0.002 \%$ for CRP and $0.003 \%$ for PCT (Table 1). Parameter A was $0.97 \%$ for CRP and $1.02 \%$ for PCT (Figure 4 ). Thus, the LOD values of IMR signals were $0.976 \%$ for CRP and $1.029 \%$ for PCT. In the logistic equation, the LOD values for assaying CRP and PCT were as low as $2.26 \times 10^{-4} \mathrm{ng} / \mathrm{mL}$ and $9.97 \times 10^{-6} \mathrm{ng} / \mathrm{mL}$, respectively.

\section{Linearity and Dynamic Range of Assaying CRP and PCT}

Figure 5 shows the results of linearity and dynamic range of assaying CRP and PCT using the IMR assay. For CRP, the IMR signals were proportional to $\phi_{\mathrm{CRP}}$ with a slope of 0.989 from 0.01 to $300 \mathrm{ng} / \mathrm{mL}$ and a coefficient of determination 
Table I The Mean Value, Standard Deviation and Coefficient of Variation of CRP and PCT

\begin{tabular}{|l|c|c|c|c|}
\hline Biomarker & $\phi(\mathbf{n g} / \mathbf{m L})$ & Mean (\%) & SD (\%) & CV (\%) \\
\hline CRP & 0 & 0.90 & 0.052 & 5.71 \\
& 0.01 & 1.01 & 0.002 & 0.21 \\
& 0.1 & 1.05 & 0.003 & 0.34 \\
& 1 & 1.17 & 0.005 & 0.40 \\
& 10 & 1.37 & 0.004 & 0.29 \\
& 100 & 1.62 & 0.003 & 0.21 \\
& 300 & 1.71 & 0.004 & 0.23 \\
& 1000 & 1.70 & 0.006 & 0.33 \\
\hline \multirow{3}{*}{ PCT } & 0 & 0.89 & 0.055 & 6.14 \\
& 0.01 & 1.08 & 0.003 & 0.25 \\
& 0.1 & 1.14 & 0.004 & 0.32 \\
& 1 & 1.23 & 0.005 & 0.41 \\
& 10 & 1.37 & 0.006 & 0.42 \\
& 50 & 1.50 & 0.005 & 0.36 \\
& 100 & 1.57 & 0.005 & 0.30 \\
& 500 & 1.71 & 0.009 & 0.51 \\
\hline
\end{tabular}

Abbreviations: CRP, C-reactive protein; PCT, procalcitonin; IMR, immunomagnetic reduction; SD, standard deviation; CV, coefficient of variation.

$\left(R^{2}\right)$ of 0.999 (Figure 5A). For PCT, the IMR signals were proportional to $\phi_{\mathrm{PCT}}$ with a slope of 1.06 from 0.1 to $100 \mathrm{ng} /$ $\mathrm{mL}\left(R^{2}=0.999\right)$ (Figure 5B). The requirement for determining the range of linearity in terms of CRP (or PCT) concentration was that the slope existed between 0.9 and 1.1. Hence, the dynamic ranges of assaying CRP and PCT were 0.01 to $300 \mathrm{ng} / \mathrm{mL}$ and 0.1 to $100 \mathrm{ng} / \mathrm{mL}$, respectively.

\section{Analysis of the Clinical Samples}

Thirty-five samples from cervicovaginal secretions of pregnant women with PPROM were obtained in this study. The median

A

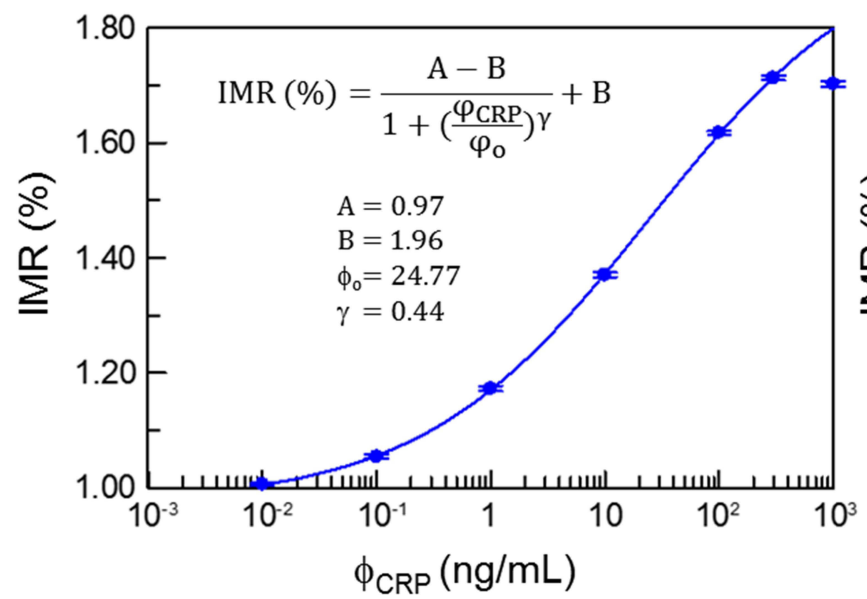

(interquartile range) gestational age at admission was 30.86 (4.43) weeks (range 22.14 to 33.71 weeks), and the gestational age at delivery was 32.57 (4.68) weeks (range 22.29 to 39.57 weeks). Figure 6 shows the individual relationships among PCT, CRP and WBC. Only a moderate correlation was noted between PCT and CRP levels in the cervicovaginal secretions $(r=0.656)$ (Figure 6A), and there were no significant correlations in the levels of PCT, CRP and WBC between cervicovaginal secretions and maternal serum (Figure 6B to 6E).

Eleven of the 35 pregnant women were diagnosed with chorioamnionitis, and the relationships between CRP, PCT and WBC and chorioamnionitis are shown in Table 2. Maternal serum CRP levels were significantly increased in the women with chorioamnionitis than in those without chorioamnionitis $(9.28$ [7.62] vs 3.04 [4.02] $\mathrm{mg} / \mathrm{L}, P=$ 0.016). After excluding two neonatal deaths due to extremely preterm birth, early-onset neonatal sepsis was diagnosed in 15 of the 33 surviving neonates. Table 3 and Figure 7 show the relationships between CRP, PCT and WBC and early-onset neonatal sepsis. There was no significant relationship between cervicovaginal secretion CRP level and neonatal sepsis (Figure 7A), however cervicovaginal secretion PCT levels were significantly increased in the neonatal sepsis group than in the non-sepsis group (45.99 [78.58] vs 9.54 [13.18] ng/mL, $P=0.039$ ) (Figure 7B).

ROC curve analysis revealed a PCT cut-off level of $20.60 \mathrm{ng} / \mathrm{mL}$ to predict early-onset sepsis (Figure 8). The area under the ROC curve was 0.71 (95\% confidence interval 0.52 to $0.90, P=0.039$ ), and the corresponding sensitivity and specificity were $73.3 \%$ and $77.8 \%$, respectively.

B

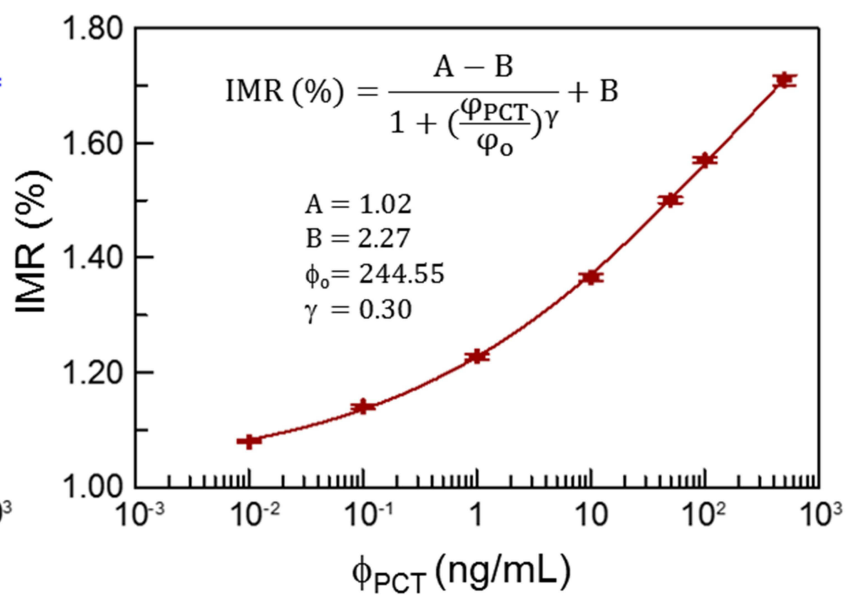

Figure 4 Calibration curves of (A) CRP and (B) PCT concentration-dependent IMR signals.

Abbreviations: CRP, C-reactive protein; PCT, procalcitonin. 
A

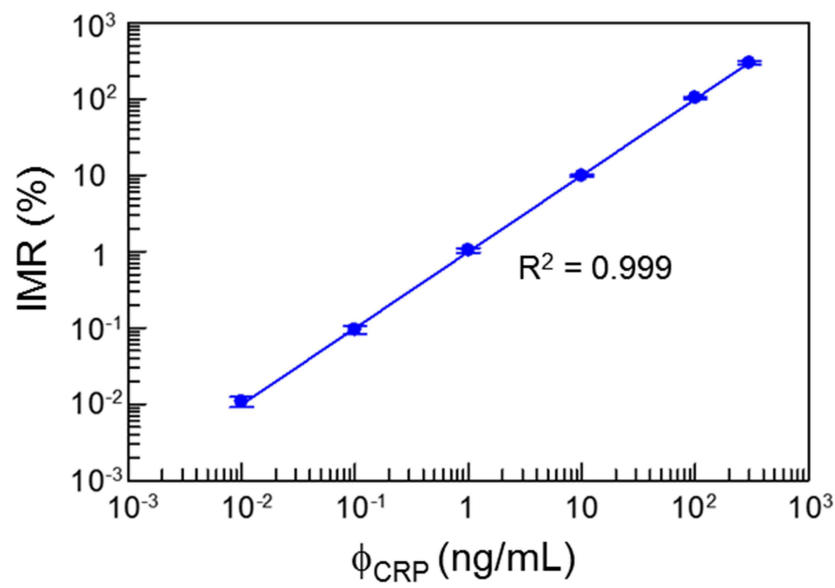

B

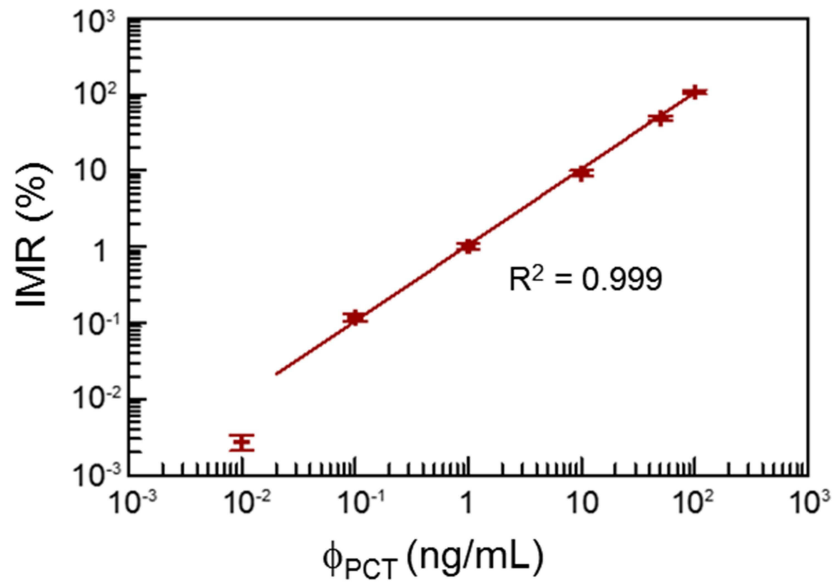

Figure 5 Linearity and dynamic range of assaying (A) CRP and (B) PCT using the IMR assay.

Abbreviations: CRP, C-reactive protein; PCT, procalcitonin.

\section{Discussion}

Early-onset sepsis is a major cause of adverse neonatal outcomes. The risk of neonatal sepsis is often assessed according to prenatal risk factors, however these factors are not sufficiently accurate. ${ }^{5}$ Thus, a practical method to predict early-onset neonatal sepsis is imperative. In this pilot study, we used an IMR assay to measure PCT and CRP concentrations in cervicovaginal secretions, and found that PCT rather than CRP was significantly increased in the mothers with PPROM and neonates with early-onset sepsis.

Few previous studies have evaluated the relationships between CRP or PCT in cervicovaginal secretions and infection. Moreover, the results of these studies have been inconsistent, and most have focused on chorioamnionitis rather than neonatal sepsis. ${ }^{17-19}$ Shim et al measured
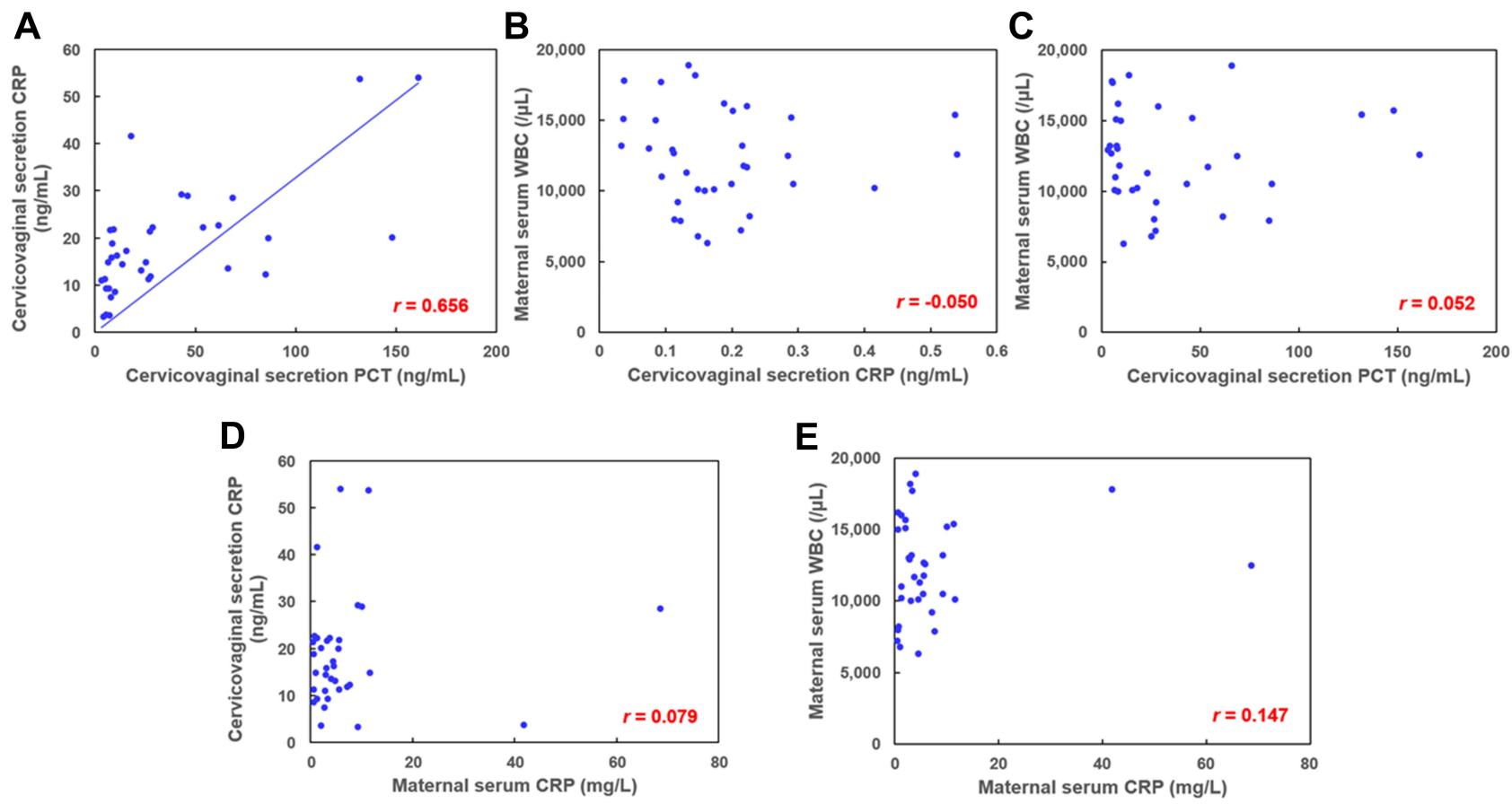

Figure 6 Individual relationships between detected biomarkers. (A) Cervicovaginal secretion PCT vs cervicovaginal secretion CRP, (B) cervicovaginal secretion CRP vs maternal serum WBC, (C) cervicovaginal secretion PCT vs maternal serum WBC, (D) maternal serum CRP vs cervicovaginal secretion CRP, and (E) maternal serum CRP vs maternal serum WBC.

Abbreviations: PCT, procalcitonin; CRP, C-reactive protein; WBC, white blood cell. 
Table 2 Relationships Between Biomarkers and Chorioamnionitis

\begin{tabular}{|l|c|c|c|}
\hline Biomarker & Without Chorioamnionitis (N = 24) & With Chorioamnionitis (N = II) & P value \\
\hline Cervicovaginal secretion CRP $(\mathrm{ng} / \mathrm{mL})$ & $16.08(10.32)$ & $14.82(20.45)$ & 0.972 \\
Cervicovaginal secretion PCT $(\mathrm{ng} / \mathrm{mL})$ & $15.92(31.88)$ & $26.72(61.88)$ & 0.500 \\
Maternal serum CRP $(\mathrm{mg} / \mathrm{L})$ & $3.04(4.02)$ & $9.28(7.62)$ & $0.016^{*}$ \\
Maternal serum WBC $(/ \mu \mathrm{L})$ & $\mathrm{II}, 500(5225)$ & $13,200(5300)$ & 0.220 \\
\hline
\end{tabular}

Notes: Continuous variables are analyzed by Mann-Whitney $U$-test and results are presented as median (interquartile range). $* P<0.05$ was considered to be statistically significant.

Abbreviations: CRP, C-reactive protein; PCT, procalcitonin; WBC, white blood cell.

Table 3 Relationships Between Biomarkers and Early-Onset Neonatal Sepsis

\begin{tabular}{|l|c|c|c|}
\hline Biomarker & Without Neonatal Sepsis (N = 18) & With Neonatal Sepsis (N = I5) & $P$ value \\
\hline Cervicovaginal secretion CRP $(\mathrm{ng} / \mathrm{mL})$ & $15.19(10.80)$ & $20.11(17.14)$ & 0.262 \\
Cervicovaginal secretion PCT $(\mathrm{ng} / \mathrm{mL})$ & $9.54(13.18)$ & $45.99(78.58)$ & $0.039 *$ \\
Maternal serum CRP $(\mathrm{mg} / \mathrm{L})$ & $3.18(3.53)$ & $5.41(9.38)$ & 0.073 \\
Maternal serum WBC $(/ \mu \mathrm{L})$ & $12,250(5300)$ & $12,600(4900)$ & 0.704 \\
\hline
\end{tabular}

Notes: Continuous variables are analyzed by Mann-Whitney $U$-test and results are presented as median (interquartile range). $* P<0.05$ was considered to be statistically significant.

Abbreviations: CRP, C-reactive protein; PCT, procalcitonin; WBC, white blood cell.

CRP concentrations in cervicovaginal secretions using ELISA, and found that an elevated level of CRP was a risk factor for chorioamnionitis in women with PPROM. ${ }^{17}$ In addition, Torbé et al measured PCT concentrations in cervicovaginal secretions using an immunoluminometric assay, and found that PCT concentration was not significantly associated with histological chorioamnionitis or neonatal congenital infections in women with PPROM. ${ }^{18}$ However, Kuyumcuoglu et al reported that PCT concentration in cervicovaginal secretions could help diagnose PPROM in patients suspected of having an intrauterine infection. ${ }^{19}$ Other studies have measured CRP or PCT concentrations in amniotic fluid using ELISA, but found that they were not useful markers for predicting infection. ${ }^{23-26}$

PCT is a 116-amino acid peptide consisting of an amino terminus, immature calcitonin and calcitonin carboxyl-terminus peptide- $1 .{ }^{27}$ The $\mathrm{N}$-terminal dipeptide of PCT can be cleaved by dipeptidyl peptidase-IV (DPIV) into an amino-terminal truncated form of procalcitonin with a peptide of 114 amino acids, which is produced earlier than non-cleaved PCT after acute systemic inflammation and is the main molecule detected during sepsis. $^{28,29}$ DPIV is a major microvillus-membrane protein, which is expressed at exceptionally high levels in the kidneys. ${ }^{30}$ Because the main source of amniotic fluid is derived from fetal urine, we hypothesize that the main biomarker detected in the cervicovaginal secretions was truncated PCT rather than full-length PCT generated by the infected fetus, and this may explain our finding that the PCT concentration in the cervicovaginal secretions was significantly increased in early-onset neonatal sepsis. Further quantitative analysis such as mass spectrometry is needed to verify our hypothesis.

Conventional sandwich assay technologies (such as ELISA) require the use of antibody pairs including capture and detection antibodies. ${ }^{31}$ As a protein (such as PCT) is truncated, the epitopes against the capture antibody and detection antibody may be in two separate segments. Although the segment with the epitope against the capture antibody may be caught by the capture antibody, the caught segment cannot bind with the detection antibody. Thus, a truncated protein cannot be efficiently detected quantitatively using sandwich assay technologies, and the concentrations of truncated proteins are frequently underestimated when using conventional methods. In the IMR assay, a single antibody is bound on the surface of the magnetic nanoparticle, and the truncated segment still has an epitope against the antibody. Hence, truncated PCT can be well assayed using the IMR assay. This also explains why the PCT concentrations in the cervicovaginal secretions measured by the IMR assay were much higher than in previous studies using conventional methods. ${ }^{18,19}$

CRP is a pentameric protein comprised of five identical non-covalently connected subunits, and acidic $\mathrm{pH}$ can modify the pentameric structure resulting in aggregation 

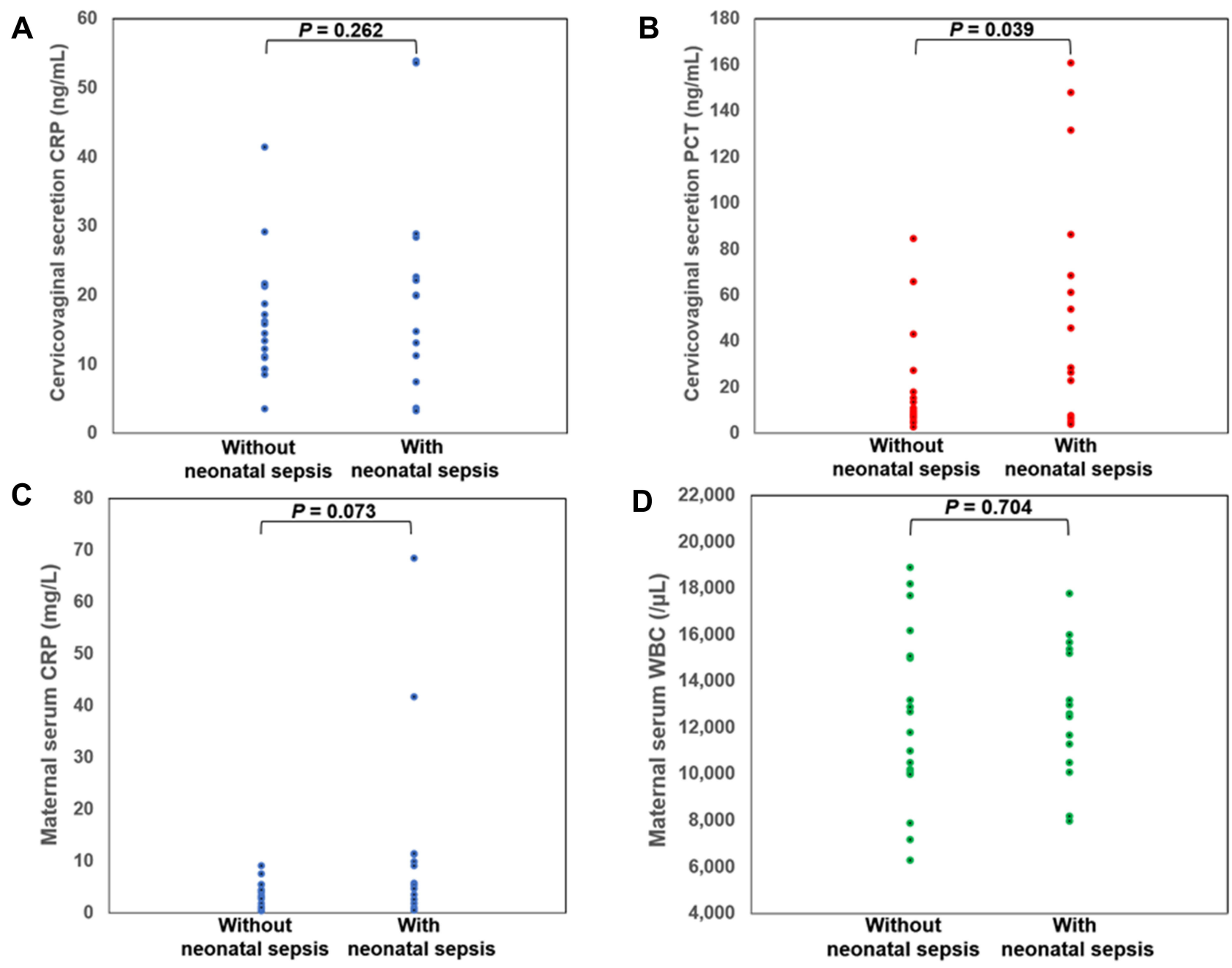

Figure 7 Relationships between various biomarkers and early-onset neonatal sepsis. (A) Cervicovaginal secretion CRP, (B) cervicovaginal secretion PCT, (C) maternal serum CRP, and (D) maternal serum WBC.

Abbreviations: CRP, C-reactive protein; PCT, procalcitonin; WBC, white blood cell.

of CRP. ${ }^{32,33}$ When CRP aggregates, it may obscure some epitopes, which may lead to partial or no detection of the epitope region in conventional sandwich assay technologies. $^{31}$ The IMR assay only needs one epitope, so it can be used to efficiently assay aggregated CRP. As a normal vaginal $\mathrm{pH}$ is between 3.8 and 4.5 , the IMR assay is an ideal method to detect CRP in an acidic environment. In this study, although a moderate correlation was noted between PCT and CRP levels in cervicovaginal secretions, we did not find a significant increase in CRP level in cervicovaginal secretions in the early-onset neonatal sepsis group. Since CRP is primarily expressed in the liver rather than in the kidneys, the relationship between cervicovaginal secretion CRP level and neonatal sepsis was not as positive as PCT. In addition, we also found that maternal serum CRP levels were significantly increased in the chorioamnionitis group, which is consistent with previous studies. $^{34,35}$

In this study, there were no significant correlations of the studied biomarkers between cervicovaginal secretions and maternal serum. Maternal serum CRP levels were associated with chorioamnionitis, and cervicovaginal secretion PCT levels were associated with neonatal sepsis. These findings imply that biomarkers in amniotic fluid have different origins of production to maternal serum, with dissimilar pathways of infection. This information may help to develop intervention strategies targeting the fetus rather than just focusing on infectious events in the mother.

In a clinical setting, if the cervicovaginal secretion PCT level is relatively low $(<20.60 \mathrm{ng} / \mathrm{mL})$, expectant management can be used if there are no other signs of 


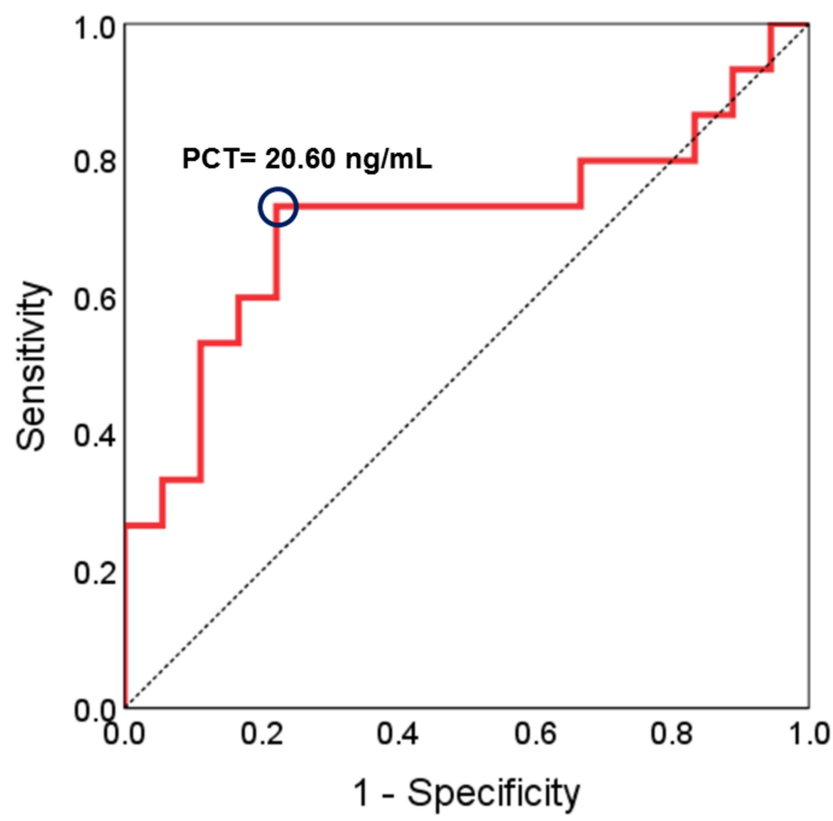

Figure 8 ROC curve of PCT for predicting early-onset neonatal sepsis using the IMR assay. The calculated cut-off PCT level was $20.60 \mathrm{ng} / \mathrm{mL}$, and the area under the ROC curve was 0.71 .

Abbreviations: ROC, receiver operating characteristic; PCT, procalcitonin.

infection. On the other hand, if the cervicovaginal secretion PCT level is relatively high ( $\geq 20.60 \mathrm{ng} / \mathrm{mL}$ ), proceeding toward delivery should be considered to prevent further damage to vulnerable preterm neonates with the presentation of early-onset neonatal sepsis. However, four of 15 fetuses with neonatal sepsis could not be detected using a cut-off PCT level of $20.60 \mathrm{ng} / \mathrm{mL}$ in this study. Since lower PCT levels in a clinical setting cannot exclude all cases of early-onset neonatal sepsis, thorough physical examinations and laboratory investigations are important for neonates born to mothers with PPROM.

Many new biomarker detection techniques have become available in recent years, such as surface plasmon resonance, quartz crystal microbalance, and electrochemical measurements. ${ }^{7}$ The LODs of surface plasmon resonance, quartz crystal microbalance and electrochemical measurements are measured in the range of $n g / \mathrm{mL}$ and $\mathrm{pg} / \mathrm{mL},{ }^{36}$ whereas the LOD of the IMR assay is measured in $\mathrm{pg} / \mathrm{mL}$ to $\mathrm{fg} / \mathrm{mL}$ (the LOD values for assaying CRP and PCT were $2.26 \times 10^{-4} \mathrm{ng} / \mathrm{mL}$ and $9.97 \times 10^{-6} \mathrm{ng} / \mathrm{mL}$ in this study). Moreover, the IMR assay can measure dozens of samples at the same time, and pre-processing for the IMR method is simple and only requires mixing the sample with the appropriate reagent before assaying.

Although there are various advantages to measuring CRP and PCT in cervicovaginal secretions using the
IMR assay, our study still has several limitations. First, we lacked data of maternal serum PCT, which is not reimbursed by the National Health Insurance system in Taiwan, and thus we could not compare the PCT levels between maternal serum and cervicovaginal secretions. Second, we did not measure the levels of CRP and PCT in cervicovaginal secretions by ELISA as a comparison with the IMR assay. In our previous study, we demonstrated that detecting biomarkers in cervicovaginal secretions with an IMR assay had a lower LOD and better sensitivity and specificity compared with ELISA. ${ }^{15}$ Third, we did not perform interference testing in this study. According to previous studies, no detectable interference effect was noted with the IMR assay. ${ }^{37,38}$ Interference of the sample color may affect the absorption of fluorescent markers in ELISA, but it does not interfere with the IMR assay because the magnetic signals are transparent. Moreover, the nonspecific molecular binding force is weaker and may be disrupted at the higher frequency rotation of magnetic nanoparticles. Fourth, the routine use of prophylactic antibiotics in mothers with PPROM may have affected the diagnosis of early-onset neonatal sepsis. Finally, the number of clinical samples in this study was limited, and further prospective randomized trials are necessary to verify our preliminary results.

\section{Conclusion}

In this pilot study, we used an IMR assay to measure CRP and PCT concentrations in cervicovaginal secretions, and found that PCT could predict early-onset sepsis in neonates born to mothers with PPROM. Understanding fetalderived infectious signals in PPROM may help to identify meaningful biomarkers related to early-onset sepsis. The early identification of neonates with a high risk of earlyonset sepsis could help neonatologists to promptly initiate antibiotic treatment to decrease the severity of sepsis, and also reduce unnecessary antibiotic exposure in neonates with a low risk of early-onset sepsis to avoid antibiotic resistance.

\section{Acknowledgments}

This work is supported by Mackay Memorial Hospital (MMH-106-71) and the Ministry of Science and Technology of Taiwan (MOST-109-2314-B-195-012MY3). We thank Mr. Yu-Hong Liu for designing the graphics of magnetic nanoparticles. 


\section{Disclosure}

The authors report no conflicts of interest in this work.

\section{References}

1. The American College of Obstetricians and Gynecologists. prelabor rupture of membranes: acog practice bulletin, number 217. Obstet Gynecol. 2020;135(3):e80-e97. doi:10.1097/AOG.0000000000003700.

2. Ramsey PS, Lieman JM, Brumfield CG, et al. Chorioamnionitis increases neonatal morbidity in pregnancies complicated by preterm premature rupture of membranes. Am J Obstet Gynecol. 2005;192 (4):1162-1166. doi:10.1016/j.ajog.2004.11.035

3. Beck C, Gallagher K, Taylor LA, et al. Chorioamnionitis and risk for maternal and neonatal sepsis: a systematic review and meta-analysis. Obstet Gynecol. 2021;137(6):1007-1022. doi:10.1097/AOG.0 000000000004377

4. Committee on Obstetric Practice. The American College of Obstetricians and Gynecologists. Committee Opinion No. 712: intrapartum management of intraamniotic infection. Obstet Gynecol. 2017;130(2):e95-e101. doi:10.1097/AOG.00000000000002236.

5. Polin RA, Papile L-A, Baley JE; Committee on Fetus and Newborn. Management of neonates with suspected or proven early-onset bacterial sepsis. Pediatrics. 2012;129(5):1006-1015. doi:10.1542/ peds.2012-0541

6. Chauhan N, Tiwari S, Jain U. Potential biomarkers for effective screening of neonatal sepsis infections: an overview. Microb Pathog. 2017;107:234-242. doi:10.1016/j.micpath.2017.03.042

7. Balayan S, Chauhan N, Chandra R, Kuchhal NK, Jain U. Recent advances in developing biosensing based platforms for neonatal sepsis. Biosens Bioelectron. 2020;169:112552. doi:10.1016/j. bios.2020.112552

8. Sproston NR, Ashworth JJ. Role of C-reactive protein at sites of inflammation and infection. Front Immunol. 2018;9:754. doi:10.3389/fimmu.2018.00754

9. Gilbert DN. Use of plasma procalcitonin levels as an adjunct to clinical microbiology. J Clin Microbiol. 2010;48(7):2325-2329. doi:10.1128/JCM.00655-10

10. Mangogna A, Agostinis C, Ricci G, et al. Overview of procalcitonin in pregnancy and in pre-eclampsia. Clin Exp Immunol. 2019;198 (1):37-46. doi:10.1111/cei.13311

11. Ryu JA, Yang JH, Lee D, et al. Clinical usefulness of procalcitonin and C-reactive protein as outcome predictors in critically ill patients with severe sepsis and septic shock. PLoS One. 2015;10(9): e0138150. doi:10.1371/journal.pone. 0138150

12. Samsudin I, Vasikaran SD. Clinical utility and measurement of procalcitonin. Clin Biochem Rev. 2017;38(2):59-68.

13. Chen CY, Hwu YM, Chen CP, et al. Quantitative analysis of total $\beta$-subunit of human chorionic gonadotropin concentration in urine by immunomagnetic reduction to assist in the diagnosis of ectopic pregnancy. Int J Nanomedicine. 2015;10:2475-2483. doi:10.2147/IJN.S81201

14. Chen CY, Chang CC, Lin CW. Clinical application of immunomagnetic reduction for quantitative measurement of insulin-like growth factor binding protein-1 in the prediction of pregnant women with preterm premature rupture of membranes. Clin Chim Acta. 2015;438:337-341. doi:10.1016/j.cca.2014.09.023

15. Wong $\mathrm{CH}$, Chen $\mathrm{CP}$, Chang $\mathrm{CC}$, et al. Bio-functionalized magnetic nanoparticles for the immunoassay of fetal fibronectin: a feasibility study for the prediction of preterm birth. Sci Rep. 2017;7(1):42461. doi:10.1038/srep42461

16. Chen CY, Hwu YM, Weng YW, et al. Clinical application of immunomagnetic reduction for quantitative analysis of beta-subunit of human chorionic gonadotropin in blastocyst culture media to differentiate embryo quality. Clin Chim Acta. 2019;491:46-51. doi:10.1016/j.cca.2019.01.012
17. Shim SS, Romero R, Jun JK, et al. C-reactive protein concentration in vaginal fluid as a marker for intra-amniotic inflammation/infection in preterm premature rupture of membranes. J Matern Fetal Neonatal Med. 2005;18(6):417-422. doi:10.1080/14786430500362231

18. Torbé A, Czajka R. Are vaginal fluid procalcitonin levels useful for the prediction of subclinial infection in patients with preterm premature rupture of membranes? J Obstet Gynaecol Res. 2005;31 (5):464-470. doi:10.1111/j.1447-0756.2005.00321.x

19. Kuyumcuoglu U, Kangal K, Guzel AI, et al. Clinical significance of procalcitonin in cervico-vaginal secretions of women with preterm rupture of membranes. Clin Exp Obstet Gynecol. 2010;37 (4):319-321.

20. Klingenberg C, Kornelisse RF, Buonocore G, et al. Culture-negative early-onset neonatal sepsis - at the crossroad between efficient sepsis care and antimicrobial stewardship. Front Pediatr. 2018;6:285. doi:10.3389/fped.2018.00285

21. Tita AT, Andrews WW. Diagnosis and management of clinical chorioamnionitis. Clin Perinatol. 2010;37(2):339-354. doi:10.1016/ j.clp.2010.02.003

22. Holzman C, Lin X, Senagore P, Chung H. Histologic chorioamnionitis and preterm delivery. Am J Epidemiol. 2007;166(7):786-794. doi:10.1093/aje/kwm168

23. Liu Y, Liu Y, Du C, et al. Diagnostic value of amniotic fluid inflammatory biomarkers for subclinical chorioamnionitis. Int $J$ Gynaecol Obstet. 2016;134(2):160-164. doi:10.1016/j.ijgo.2016.01.007

24. Marchocki Z, Vinturache A, Collins K, et al. Amniotic fluid C-reactive protein as a predictor of infection in caesarean section: a feasibility study. Sci Rep. 2018;8(1):6372. doi:10.1038/s41598-018-24569-8

25. Dulay AT, Buhimschi IA, Zhao G, et al. Compartmentalization of acute phase reactants interleukin-6, C-reactive protein and procalcitonin as biomarkers of intra-amniotic infection and chorioamnionitis. Cytokine. 2015;76(2):236-243. doi:10.1016/j.cyto.2015.04.014

26. Horinouchi T, Yoshizato T, Kozuma Y, et al. Prediction of histological chorioamnionitis and neonatal and infantile outcomes using procalcitonin in the umbilical cord blood and amniotic fluid at birth. J Obstet Gynaecol Res. 2018;44(4):630-636. doi:10.1111/jog.13573

27. Schneider HG, Lam QT. Procalcitonin for the clinical laboratory: a review. Pathology. 2007;39(4):383-390. doi:10.1080/003130 20701444564

28. Struck J, Strebelow M, Tietz S, et al. Method for the selective measurement of amino-terminal variants of procalcitonin. Clin Chem. 2009;55(9):1672-1679. doi:10.1373/clinchem.2008.123018

29. Weglöhner W, Struck J, Fischer-Schulz C, et al. Isolation and characterization of serum procalcitonin from patients with sepsis. Peptides. 2001;22(12):2099-2103. doi:10.1016/s0196-9781(01) 00541-1

30. Kenny AJ, Booth AG, George SG, et al. Dipeptidyl peptidase IV, a kidney brush-border serine peptidase. Biochem J. 1976;157 (1):169-182. doi:10.1042/bj1570169

31. Itoh K, Suzuki T. Antibody-guided selection using capture-sandwich ELISA. Methods Mol Biol. 2002;178:195-199. doi:10.1385/1-59259240-6:195

32. Blizniukov OP, Kozmin LD, Falikova VV, et al. Aggregation of C-reactive protein in solutions at acid pH. Biofizika. 2003;48 (5):844-852.

33. Hammond DJ Jr, Singh SK, Thompson JA, et al. Identification of acidic $\mathrm{pH}$-dependent ligands of pentameric C-reactive protein. $J$ Biol Chem. 2010;285:36235-36244. doi:10.1074/jbc.M110.142026

34. Musilova I, Kacerovsky M, Stepan M, et al. Maternal serum C-reactive protein concentration and intra-amniotic inflammation in women with preterm prelabor rupture of membranes. PLoS One. 2017;12:e0182731. doi:10.1371/journal.pone.0182731

35. Asadi N, Faraji A, Keshavarzi A, et al. Predictive value of procalcitonin, C-reactive protein, and white blood cells for chorioamnionitis among women with preterm premature rupture of membranes. Int J Gynaecol Obstet. 2019;147(1):83-88. doi:10.1002/ijgo.12907 
36. Alvarez M, Lechuga LM. Microcantilever-based platforms as biosensing tools. Analyst. 2010;135(5):827-836. doi:10.1039/b908503n

37. Huang KW, Yang SY, Hong YW, et al. Feasibility studies for assaying alpha-fetoprotein using antibody-activated magnetic nanoparticles. Int J Nanomedicine. 2012;7:1991-1996. doi:10.2147/ IJN.S28245
38. Chang CH, Lai ZX, Lin HL, et al. Use of immunomagnetic reduction for C-reactive protein assay in clinical samples. Int J Nanomedicine. 2012;7:4335-4340. doi:10.2147/IJN.S31030

\section{Publish your work in this journal}

The International Journal of Nanomedicine is an international, peerreviewed journal focusing on the application of nanotechnology in diagnostics, therapeutics, and drug delivery systems throughout the biomedical field. This journal is indexed on PubMed Central, MedLine, CAS, SciSearch ${ }^{\mathbb{B}}$, Current Contents ${ }^{\mathbb{B}} /$ Clinical Medicine, $^{2}$
Journal Citation Reports/Science Edition, EMBase, Scopus and the Elsevier Bibliographic databases. The manuscript management system is completely online and includes a very quick and fair peer-review system, which is all easy to use. Visit http://www.dovepress.com/ testimonials.php to read real quotes from published authors. 